\title{
tic\&société
}

Vol. 5, n²-3 | 2e sem. 2011 / 1er sem. 2012

Les TICs dans les pays des Suds

\section{Technologies éducatives et développement : une brève histoire de la télévision scolaire au Niger}

Gado Alzouma

\section{(2) OpenEdition \\ Journals}

Édition électronique

URL : http://journals.openedition.org/ticetsociete/1025

DOI : 10.4000/ticetsociete. 1025

Éditeur

Association ARTIC

Référence électronique

Gado Alzouma, «Technologies éducatives et développement : une brève histoire de la télévision

scolaire au Niger », tic\&société [En ligne], Vol. 5, n²-3| 2e sem. 2011 / 1er sem. 2012, mis en ligne le

18 juin 2012, consulté le 19 avril 2019. URL : http://journals.openedition.org/ticetsociete/1025 ; DOI :

10.4000/ticetsociete.1025 
tic\&société - 5 (2-3), $2^{\text {ème }}$ semestre 2011 - $1^{\text {er }}$ semestre 2012

\title{
Technologies éducatives et développement : une brève histoire de la télévision scolaire au Niger
}

\author{
Gado ALZOUMA, \\ Associate Professor of Anthropology \\ School of Arts and Science \\ American University of Nigeria \\ Lamido Zubairu Way, Yola By-Pass, PMB 2255, Yola, Adamawa State, \\ Nigeria
}

Alzouma@aun.edu.ng

Gado Alzouma est maître de conférences à l'Université Américaine du Nigéria. II a fait ses études supérieures en France (Bordeaux et Strasbourg) et aux Etats-Unis (Carbondale, Illinois). II est titulaire d'un Ph.D. en anthropologie de la Southern Illinois University, USA. Avant de rejoindre I'UAN, il a enseigné pendant plusieurs années à l'Université Abdou Moumouni de Niamey, au Niger. II a également occupé le poste de coordinateur, Evaluation et Systèmes d'Apprentissage du Programme " Communautés Africaines et Société de I'Information " (Acacia) à Dakar, au Sénégal pour le compte du Centre de Recherche pour le Développement International (CRDI, Ottawa, Canada). II a aussi enseigné à la Southern Illinois University de Carbondale aux Etats-Unis et y a travaillé en tant que chercheur associé (research fellow) au Global Media Research Center. Ses travaux portent sur les technologies de l'information et de la communication pour le développement, sur les usages sociaux des objets techniques et sur les inégalités. II est l'auteur de nombreux articles dans des revues académiques internationalement reconnues. 
Technologies éducatives et développement :

une brève histoire de la télévision scolaire au Niger

\title{
Technologies éducatives et développement : une brève histoire de la télévision scolaire au Niger
}

\begin{abstract}
Résumé
L'histoire du développement en Afrique est jalonnée de toutes sortes d'expériences et de projets centrés autour de nouvelles technologies souvent présentées comme des solutions miracles aux problèmes de santé, d'éducation, ou de production agricole. L'échec de certains de ces projets permet de montrer les limites de l'idéologie technocentriste qui a aujourd'hui refait surface dans le discours développementaliste et qui fait reposer sur la technologie - et la technologie seule - le développement social et économique du continent africain. C'est le cas de l'expérience de la télévision scolaire au Niger qui, entamée en 1964 et abandonnée en 1979, devait assurer un accès rapide à la scolarisation pour tous les enfants en âge d'aller à l'école mais qui n'a pas réussi à transformer le système éducatif ou à relever de façon significative le taux de scolarisation qui, quarante-six ans plus tard, est toujours en dessous de $60 \%$. Cet article se propose de montrer que cette expérience n'a pas été un " échec " dû à une déficience de participation communautaire, comme l'avancent généralement les évaluations de projets, mais le résultat d'une confrontation de visions divergentes du monde et de la société, la télévision scolaire étant devenue un enjeu de lutte à la fois sociale et politique qui s'est soldé par la victoire de l'une des parties et l'affectation de la télévision éducative à des fins autres que celles pour lesquelles elle avait été conçue.
\end{abstract}

Mots-clés: Technologies éducatives; développement; télévision; Niger. Educational Technologies ; Development ; Televison ; Niger.

\begin{abstract}
The history of development in Africa is littered with all sorts of experiments and projects centred on new technologies often presented as a panacea to the problems of health, education, or agricultural production. The failure of some of those projects shows the limits of the technocentrist approach which
\end{abstract}


has recently resurfaced in the developmentalist discourse and which is based on the idea that technology (and technology alone) can solve all the social and economic problems Africa is facing. This is the case of the educational television in Niger that was supposed to ensure a rapid access to universal education. It started in 1964 and was abandoned in 1979. It failed to transform the educational system in any significant way, the school enrolment rate in Niger still being under $60 \%, 46$ years after the experiment started. In this paper, I intend to show that this failure was not due to a lack of community involvement as generally advanced by project evaluations, but the result of a confrontation of divergent views of the world and society. Indeed, the educational television has become an issue of both social and political struggle, which resulted in the victory of one of the parties and the allocation of the educational television to other purposes for which it was not previously designed.

Key words : educational technologies; development; television; Niger. Technologies éducatives; développement; télévision; Niger.

\section{Resumen}

La historia del desarrollo en África está llena de toda clase de experimentos y proyectos centrados en las nuevas tecnologías a menudo se presentan como soluciones milagrosas a los problemas de salud, educación, o la producción agrícola. El hecho de que algunos de estos proyectos han fracasado nos permite mostrar los límites de la ideología tecnocéntrica que ha resurgido en el discurso desarrollista de hoy y que se basa en la tecnología (y la tecnología por sí sola), el desarrollo social y económico de África. Este es el caso de la experiencia de la televisión educativa en Níger, que comenzó en 1964 y fue abandonado en 1979, que debe garantizar el acceso oportuno a la educación para todos los niños de edad asisten a la escuela, pero no ha tenido éxito transformar el sistema educativo o para elevar significativamente la tasa de matrícula que, cuarenta y seis años tarde sigue siendo inferior al $60 \%$. Este artículo pretende mostrar que esta experiencia no fue un fracaso debido a la falta de participación de la comunidad como se argumentó en general, las evaluaciones de proyectos, sino el resultado de una confrontación visiones divergentes del mundo y la sociedad. La televisión educativa se ha convertido en un reto para luchar social y política que se tradujo en la victoria de una de las partes y la asignación de la televisión educativa para fines distintos de aquellos para los que fue diseñado.

Palabras clave: Tecnologías para la Educación, el desarrollo, la televisión del Níger. 
Technologies éducatives et développement :

une brève histoire de la télévision scolaire au Niger

\section{Introduction}

La plupart des idées et concepts qui ont trait au développement et qui, à un moment ou à un autre, sont en vogue en Afrique ou dans les pays en développement, trouvent leurs origines dans des préoccupations et des débats ayant cours en Occident. Ce sont presque toujours des entrepreneurs sociaux européens (souvent des hommes pleins de bonne volonté, d'abnégation et d'imagination qui ont décidé de se mettre au service des autres parce qu'ils croient profondément qu'il est possible de changer les choses) qui font de l'Afrique la terre d'excellence pour la réalisation des idées dont ils sont porteurs. Ces idées ne sont pas toujours utopiques au sens populaire du terme, c'est-àdire que ce ne sont pas des projets déraisonnables ou irréalisables. Elles sont parfois réalistes et réalisables. Certaines d'entre elles correspondent aussi à des besoins réels et parfaitement identifiés et sont parfois des solutions originales et même efficientes lorsqu'appliquées. Elles bénéficient également de concepteurs et de soutiens multiformes, presque toujours enthousiastes et déterminés et soucieux d'impliquer ou d'associer les communautés concernées à la réalisation de leurs objectifs. Leur faisabilité et leur mise en œuvre ont aussi été souvent soigneusement étudiées et les technologies adaptées aux contextes sociaux et aux réalités culturelles et économiques. Et, pourtant, elles finissent presque toujours par échouer, par être abandonnées ou condamnées à ne jamais dépasser le stade expérimental, anecdotique ou, au mieux, par se réduire à des activités qui se poursuivent au long de plusieurs décennies sans pouvoir gagner une adhésion suffisamment large pour être considérées comme une composante significative du système d'ensemble.

Certaines de ces idées naissent autour d'innovations technologiques pour lesquelles des agents du développement, des éducateurs et des décideurs économiques perçoivent des applications utiles dans de nouveaux contextes sociaux et culturels. Elles sont parfois guidées par une sorte de messianisme technologique mis au service du développement économique et social.

En Afrique et ailleurs dans le monde, c'est le secteur de l'éducation qui a souvent servi de terrain d'expérimentation pour la mise en œuvre de ces projets. L'un des tout derniers exemples dans ce domaine est l'école numérique de brousse dont l'objectif est d'installer des tableaux numériques interactifs dans les écoles africaines [Marie, 2009]. Vingt-six tableaux numériques interactifs ont ainsi été placés en Afrique dans cinq pays : Bénin, Burkina Faso, Mali, Niger et Sénégal. Parmi ces pays, le Sénégal et le Niger correspondent aussi, comme 
nous le verrons plus loin, à ceux dans lesquels ont été implantées les premières télévisions scolaires. Tout comme pour ces-dernières d'ailleurs, le projet d'écoles numériques de brousse vise à atteindre l'objectif d'une scolarisation de tous les enfants en âge d'aller à l'école. Les écoles numériques de brousse ne sont pas les seuls projets éducatifs centrés autour des TIC en Afrique. Au Niger, il existe un Projet d'alphabétisation de base par téléphone cellulaire (Projet $A B C, 123)$ dont les initiateurs sont Catholic Relief Services et les très sérieuses Tufts University des États-Unis et Oxford University de Grande-Bretagne. Le Projet ABC, 123 utilise les téléphones mobiles pour la promotion de l'alphabétisation des adultes. II existe aujourd'hui dans plus de 110 villages dans les régions de Dosso et de Zinder [Lybert, Acker et Ksoll, 2010]. D'après les premiers résultats d'une évaluation menée par les responsables du projet, en comparaison des méthodes traditionnelles d'alphabétisation, le téléphone mobile est une méthode efficace pour élever les performances des apprenants aux tests [Lybert, Acker et Ksoll, 2010].

Les écoles numériques de brousse et les projets d'alphabétisation par téléphone portable ne sont, en réalité, que les derniers d'une longue série de projets éducatifs centrés sur les TIC en Afrique, qui ont été mis en œuvre sur le continent depuis la fin des années 1990. L'un d'entre eux, l'université virtuelle africaine, vise à donner aux étudiants africains une "éducation de classe mondiale " sans qu'ils aient besoin de quitter leurs pays, à travers l'éducation à distance et l'e-learning (l'éducation au moyen des technologies de l'information et de la communication). Créée à l'initiative de cinq pays africains (Kenya, Sénégal, Mauritanie, Mali et Côte d'Ivoire) en 1997, l'African Virtual University (AVU) clame avoir formé plus de 40000 étudiants à ce jour (AVU portal). Son pendant francophone, l'Université virtuelle francophone (UVF), à laquelle on peut ajouter les campus numériques francophones, fonctionnent aussi sur le même principe et ont formé à ce jour des milliers d'étudiants. Mais tout comme les écoles numériques de brousse ou l'alphabétisation par téléphone portable, leurs activités sont largement marginales et insuffisamment intégrées aux systèmes d'enseignement nationaux. Enfin, elles sont surtout le prolongement d'expériences déjà entreprises en Occident où elles n'ont pas davantage réussi à transformer l'enseignement.

Ceci est particulièrement vrai des télévisions scolaires qui, entre 1964 et 1979, ont constitué une expérience originale (en réalité un prolongement de ce qui se faisait déjà en France) tentée au Niger pour permettre d'assurer en un temps relativement court la scolarisation totale des enfants. II s'agissait d'utiliser des messages télévisuels diffusés à raison de quatre émissions quotidiennes, soit 2840 émissions au total sur un cycle de quatre années [Egly, 1973], pour enseigner non seulement la langue française, mais aussi le calcul, les sciences 
Technologies éducatives et développement:

une brève histoire de la télévision scolaire au Niger

d'observation, l'écriture, la lecture et les travaux pratiques aux enfants. L'enseignement, assuré par des moniteurs locaux, était donné en langue française et reposait sur l'observation du milieu et l'expression libre des enfants. En 1979, date à laquelle il y fut mis fin, la télévision scolaire aura concerné 214 classes de 50 élèves en moyenne et aura employé une équipe de 135 personnes dont 55 assistants techniques français [Egly, 1986]. De l'avis de nombreux observateurs, la mise en pratique de cette idée novatrice ne fut pas uniquement négative puisque les études ont prouvé que les élèves passés par la télévision scolaire avaient une nette avance sur les autres, surtout en français. L'expérience ne fut cependant pas poursuivie et le Niger est revenu aux méthodes traditionnelles d'enseignement. La télévision scolaire nigérienne est donc généralement considérée comme un échec, notamment dans l'objectif de scolarisation totale des enfants nigériens qui lui était assigné. Aujourd'hui encore, en 2011, quarante-six ans après qu'elle ait été implantée, le Niger compte un taux de scolarisation de moins de $60 \%$.

Dans l'article qui suit, nous souhaitons montrer pourquoi ce projet ambitieux, qui reposait sur l'utilisation de moyens audiovisuels pour assurer une éducation primaire totale, a été abandonné et quelles sont les leçons à en tirer pour les expériences actuelles. Toutefois, il ne s'agit pas pour nous d'entreprendre une énième critique des utopies éducatives ou de nier l'importance de la technologie pour le développement et l'éducation. II ne s'agit pas non plus de refuser l'innovation ou les expériences innovantes en matière d'éducation. Nous croyons, tout comme Max Egly, que " c'est en les taxant d'utopiques que l'on freine des recherches utiles, que l'on met fin à des entreprises novatrices, permettant ainsi aux systèmes périmés ou inadaptés de survivre " [1979, p.1]. On peut d'ailleurs affirmer que des expériences d'éducation similaires, menées à la même époque dans d'autres pays (c'est le cas du Salvador) avec la télévision, ont réussi [Mayo, 1976] et que, même au Niger, l'échec n'était pas tant pédagogique que social et économique. Nous croyons néanmoins que les espoirs de scolarisation universelle (terme consacré dans le jargon " développementaliste " pour parler de scolarisation totale des enfants d'âge scolaire d'une nation quelconque) ne sauraient reposer sur la technologie seule sans faire évoluer, dans un même mouvement, l'ensemble des agents qui concourent à l'avènement d'une école développée. Notre objectif est donc de mettre en évidence les limites des approches technocentristes du développement et les conditions sans lesquelles les expériences en matière de d'éducation, même les plus innovatrices, ne peuvent réussir.

Dans un premier temps, nous rappellerons brièvement l'origine de la télévision scolaire et autres technologies éducatives similaires en France. Puis 
nous verrons comment ces idées, nées ailleurs, ont été transposées au Niger. La deuxième partie de notre article sera consacrée à l'examen de l'évolution de la télévision scolaire au Niger. Nous verrons ainsi comment ce qui était considéré comme un succès par les experts a fini par être abandonné par le gouvernement nigérien. Enfin, nous montrerons que cet échec, en dehors même des raisons financières, peut être expliqué comme le résultat d'une approche fondée sur le modèle diffusionniste. Le défaut principal de ce modèle est qu'il ne prend pas en compte les conditions liées à l'appropriation sociale de la technologie et néglige les " compromis » (entre agents dans leurs rapports à l'objet technique) nécessaires à l'intégration de cet objet aux normes sociales.

\section{Méthodologie}

Cet article repose d'abord sur des données qualitatives recueillies lors d'entretiens semi-directifs à Niamey, capitale du Niger, entre juin et août 2010 et de nouveau en mars 2011, auprès d'une trentaine d'anciens élèves (tous issus des promotions 1966-1972 et 1972-1978), d'une dizaine d'anciens moniteurs (enseignants), d'une dizaine de techniciens et de cinq anciens administrateurs nationaux de la télévision scolaire. Les entretiens ont été réalisés à l'aide de guides rédigés à cet effet, dont certains remplis directement par les interviewés (qui ont par la suite répondu à des questions complémentaires posées par téléphone). Notre méthode n'étant pas statistique, nous ne nous sommes pas soucié d'une représentativité au sens quantitatif du terme, mais de collecter les informations approfondies que pouvaient nous livrer nos interlocuteurs. Bien que nous ayons nous-mêmes préalablement défini les thèmes abordés, nous avons veillé à ne pas nous enfermer dans un cadre prédéfini afin de permettre à nos informateurs de retracer l'expérience telle qu'elle a été vécue et de recueillir leurs opinions et sentiments sur le projet et ses concepteurs, le contexte dans lequel sa mise en œuvre est intervenue, sa réalisation et son échec. En dehors des entretiens semi-directifs, nous avons aussi eu recours à l'analyse de données recueillies dans des journaux de l'époque, à des rapports d'évaluation, à des articles scientifiques rédigés sur l'expérience et aux statistiques disponibles.

\section{Aux origines de la télévision scolaire en France}

Comme souligné plus haut, la télévision scolaire ne fut qu'une idée appliquée d'abord en France et reprise ensuite au Niger et dans d'autres pays africains pour assurer une scolarisation totale des enfants. En France, comme ailleurs 
Technologies éducatives et développement :

une brève histoire de la télévision scolaire au Niger

dans d'autres pays occidentaux, il a toujours existé un lien étroit entre "l'évolution des technologies, des idéologies et des modes" [Duboux, 1996, p. 21], notamment en matière d'enseignement. Ainsi, aussi loin qu'on remonte dans l'histoire de l'éducation, les innovations technologiques ont toujours été transposées dans les salles de classe et celles-ci ont souvent été appelées à s'adapter à ces innovations. Dès les années 1930 par exemple, certaines classes furent dotées de ce qu'on appelait alors les «petits appareils PathéBaby capables de projeter des films à perforation centrale » [Vincent, 1981, p. 146]. Cet auteur souligne aussi qu'à la même époque plusieurs classes possédaient un phonographe qu'on remontait à la manivelle pour initier aux chants scolaires. Cependant, c'est seulement dans les années 50 que « le vote de la loi dite Barange, octroyant à chaque groupe scolaire des crédits supplémentaires destinés aux matériels d'enseignement» va permettre aux écoles "de se munir de projecteurs à vues fixes de type Larousse [...] Chaque maître pouvait illustrer, par images de grand format, toutes les leçons, les maisons d'édition rivalisant à qui mieux mieux pour fabriquer, en toutes disciplines, films et commentaires adéquats " [Vincent, 1981, p. 146]. Ce fut cependant la radio scolaire, initiée avec les encouragements de l'Unesco dès le début des années 1940, qui va être à l'origine des premières innovations devant servir de fondement à l'implantation de la télévision scolaire. La radio donnait en effet la possibilité à un seul maître d'enseigner à plusieurs milliers d'élèves en même temps grâce à des leçons préenregistrées et diffusées à heures et jours fixes sur toute l'étendue du territoire national. C'est de là que naquit l'idée qu'il était possible de réduire les coûts de l'éducation en faisant intervenir un seul maître là où il en aurait fallu des dizaines, voire des centaines. Ceci paraissait particulièrement vrai et utile pour des pays qui, comme le Niger, n'avaient pas (et n'ont toujours pas) les ressources suffisantes pour faire face aux dépenses de la formation d'un très grand nombre d'enseignants dans un contexte de croissance démographique rapide. Dans le cas de la radio scolaire en France, une telle innovation permettait même de faire face, pour les familles, aux coûts occasionnés par les déplacements, de libérer ainsi du temps pour les parents, ou de regrouper les élèves d'une même classe d'âge autour d'un poste récepteur unique. Pour les enseignants eux-mêmes, dont certains avaient des difficultés à s'exercer au chant par exemple, il était aisé de suivre les leçons de chanteurs professionnels.

À partir de 1945 et jusqu'en 1950, dans le sillage du cinéma scolaire et de la radio scolaire, "une section enseignement de la télévision nationale » va produire et diffuser des émissions scolaires [Glikman, 2010, p. 109]. Ces émissions seront ensuite confiées en 1951 à l'Institut pédagogique national (IPN) quand la télévision s'avéra incapable de les poursuivre. Les ambitions 
pédagogiques de la Radio-Télévision scolaire (RTS) n'étaient d'ailleurs pas limitées aux enfants. Comme le note Glikman, " de nombreux programmes de télévision scolaire et éducative pour adultes ont été régulièrement diffusés, des années cinquante aux années quatre-vingt, sur le réseau national hertzien de la France, qui a été l'un des précurseurs dans ce domaine. [...] Parmi ces programmes, les premières émissions de télévision éducative pour adultes, produites, à partir de 1964, à l'intention de publics peu ou moyennement scolarisés, sous l'égide du ministère de l'Éducation nationale, ont longtemps été désignées, ainsi que le service chargé de leur conception, sous le nom de RTS/Promotion » [1995, p. 63]. L'histoire de la télévision scolaire en France a donc été marquée par de nombreux changements, aussi bien dans les objectifs qui lui étaient assignés que dans les orientations philosophiques qui la soustendaient ou les noms, les structures organisationnelles et les contenus pédagogiques. Ces changements traduisent en réalité l'évolution de la société française, elle-même marquée par de profondes transformations et des besoins croissants en matière d'éducation (qu'on voulait démocratique, c'est-à-dire pour tous), notamment durant l'ère d'industrialisation et d'expansion économique connue sous le nom des Trente glorieuses (1945-1975).

Cependant, un objectif était resté toujours constant: il s'agissait de transformer non seulement l'école mais aussi la société grâce à la télévision qui allait offrir "une fenêtre sur le monde" aux élèves, favoriser l'égalité des chances et réduire les disparités scolaires et, surtout, faire bénéficier tous les élèves en même temps des cours modèles offerts par les mêmes professeurs, choisis parmi les plus éminents. Enfin, comme pour la radio scolaire, la télévision permettait de faire face au manque de professeurs qualifiés, en nombre insuffisant. Très vite cependant, elle dut faire face à des difficultés de toutes sortes : en raison de la philosophie même qui la sous-tendait (remédier à l'insuffisance du nombre d'enseignants), les émissions de la télévision éducative seront qualifiées de "palliatives" par les syndicats d'enseignants [Glikman, 1995 , p. 64] qui souvent s'y opposeront au nom du principe selon lequel on ne saurait confier une tâche aussi complexe que celle de l'enseignement (qui suppose échanges émotionnels et compréhension) à une machine [Egly, 1984] ; s'y ajouteront l'insuffisance de l'équipement, le caractère aléatoire du fonctionnement des récepteurs qui peuvent tomber en panne à tout moment sans que les maîtres aient la possibilité de faire quoi que ce soit, n'étant pas formés à réparer des récepteurs, le caractère fixe des heures de diffusion qui ne tenaient pas toujours compte de l'organisation de l'enseignement et surtout le caractère mécanique et rébarbatif des cours $^{1}$ qui, selon un présupposé courant

\footnotetext{
1 Max Egly (1973) a cependant démontré que la critique relative au caractère mécanique et rébarbatif des cours de la télévision scolaire était peu fondée. En lieu et place des images
} 
Technologies éducatives et développement :

une brève histoire de la télévision scolaire au Niger

à l'époque, ne favorisait pas l'interaction en raison même de la nature des médias. Autrement dit, ce qu'on reprochait à la télévision en général (développement d'une absorption passive et unidirectionnelle d'informations) se trouvait reproduit à l'école, menaçant gravement le développement d'un esprit critique chez les élèves. Pour toutes ces raisons, l'intégration formelle (c'est-àdire institutionnalisée) de la télévision aux programmes scolaires fut considérablement réduite en France et laissée à l'appréciation des enseignants qui, tout comme aux premières heures de l'expérience, peuvent aujourd'hui encore y avoir recours ou non sur décision personnelle.

\section{La télévision scolaire au Niger (Télé-Niger)}

Bien que le constat d'un échec relatif ait été fait au début des années 1970 en France, cela n'a pas empêché le ministère français de la Coopération d'encourager la transposition de l'expérience en Afrique. Le contexte de l'époque favorisait largement ces projets. Le début des années 1960 correspondait aux indépendances et il régnait en Afrique une certaine euphorie progressiste entretenue par toutes sortes d'idées aux accents développementalistes. Dans les cercles académiques, les organismes de développement international et les agences de l'ONU, les aspirants au rôle de réformateurs sociaux étaient légion, qui plaçaient dans la technologie l'espoir d'un décollage rapide de l'Afrique. Le continent, perçu comme une terre vierge, apparaissait comme un terrain d'expérimentation parfait pour faire accéder les Africains aux bienfaits du progrès technologique. Au cœur de ce discours développementaliste se trouvait l'idée que l'état de l'Afrique d'alors n'était pas souhaitable et devait être changé au nom du progrès et du bien-être des populations. On n'en était pas encore à l'époque où les interventions extérieures étaient critiquées au nom de la négligence des conditions sociales et culturelles et de la préservation des valeurs culturelles des sociétés indigènes. La logique top-down du développement imposé sur la base du modèle occidental n'était pas encore mise en question.

didactiques fixes, les concepteurs des émissions scolaires, qui comptaient parmi eux des experts de toute sorte (éducateurs, psychologues, ethnologues, graphistes, etc.), élaboraient celles-ci sur le modèle du "théâtre filmé » et du "mouvement ", c'est-à-dire l'originalité et la créativité en s'inspirant du cinéma de spectacle et de la vie ordinaire des élèves. En outre, les émissions, soumises à de constantes réévaluations et critiques collectivement formulées, étaient toujours réadaptées, réajustées et améliorées au vu des observations faites. Fondées sur la notion de « spectacle pédagogique », elles étaient donc loin d'être rébarbatives ou mécaniques. 
Par ailleurs, la dépendance des gouvernements africains envers la France pour tout ce qui concernait l'orientation des politiques nationales de développement était encore forte ; l'idée d'une certaine tutelle était encore largement acceptée et assumée par les agents et les décideurs, aussi bien en Afrique qu'en France. Bien entendu aussi, la coopération offerte par la France dans ce cadre n'était pas toujours dénuée d'arrière-pensées telles que l'expansion de la langue française en Afrique, comme le rapporte une responsable française de la coopération en matière d'éducation au début des années 1960, à l'époque où s'élaboraient les projets de télévision scolaire : "Un autre étonnement : ayant lu, assemblé, classé tout ce qui m'avait été fourni sur les pays nouvellement indépendants d'Afrique, où il était constamment question de développement, toutefois peu encore de développement culturel [...], j'eus la surprise d'entendre dans une réunion Jean-Pierre Dannaud, exprimer l'urgence absolue de faire comprendre aux populations l'universalité de la langue française. Que l'on ne me fasse pas de faux procès, c'était l'urgence qui me gênait, pas l'universalité. " [de Saivre, 1998, p.160]. Des idées comme celles de J.-P. Dannaud, qui était alors directeur de la Coopération culturelle, auront de fortes incidences sur l'orientation des programmes scolaires. Toutefois, on ne peut aussi s'empêcher de remarquer qu'il régnait chez les concepteurs une sorte d'esprit missionnaire, sous-tendu par le développement technologique dont il était entendu que les bienfaits devaient pouvoir bénéficier à tous et particulièrement aux populations démunies d'Afrique : "Le projet (Télé-Niger), dont le siège était à Niamey lui-même, fut passionnant. L'équipe avait décidé de centrer l'enseignement sur l'enfant africain et le milieu dans lequel il vivait, ceci en français. Il était difficile à cette époque de faire autrement et l'on se trouvait dans le pays d'un président, Diori Hamani, qui, comme on le sait, fut l'un des fondateurs de la francophonie. Le projet s'adressait à des enfants non scolarisés du milieu rural. On venait nous voir de partout [...] j'ai rarement vu un projet fonctionner avec autant d'enthousiasme et de probité intellectuelle. Nous y croyions tous, nous étions jeunes, la Coopération aussi. " [de Saivre, 1998, pp. 161-162] C'est aussi à cette époque que les grands projets d'éducation de masse furent conçus sous la houlette d'organisations et de militants tiersmondistes. À l'appel de certaines personnalités influentes comme Gaston Berger, décédé peu auparavant, les "nouvelles» technologies de la communication, qui étaient alors la radio, la télévision et les satellites, apparurent très vite comme une panacée pour le développement accéléré de l'Afrique. Aussi, à défaut de voir ceux-ci en exprimer le besoin, il fallait en convaincre les gouvernements africains :

«II y avait un autre hic mais je ne l'appris que plus tard. C'est que le Président Diori, bien que lui-même 
Technologies éducatives et développement:

une brève histoire de la télévision scolaire au Niger

très francophone, n'avait été demandeur de la téléscolaire du Niger que parce qu'on l'en avait convaincu. Qu'il ait été content que ce grand projet ait lieu chez lui est une autre affaire et il fut toujours agréable avec tous. [...] Lorsque je résidais en Afrique, j'avais l'impression que l'on traitait ces pays comme de gigantesques laboratoires où l'on essayait, si bons qu'ils fussent, des projets que l'on ne pouvait pas expérimenter en France. » [de Saivre, 1998, p.162].

La «demande » du gouvernement nigérien pour la mise en œuvre d'un projet d'éducation télévisuelle, exprimée en juin 1963, a donc été pour le moins encouragée, sinon suscitée. Elle était d'ailleurs survenue dans un contexte de réforme de l'enseignement engagée par les autorités nigériennes qui cherchaient le moyen le plus efficace et le moins onéreux d'assurer une scolarisation totale des enfants nigériens. Pour la plupart des agents de développement de l'époque, la télévision scolaire offrait cette opportunité. Un accord fut donc conclu avec la France pour un projet qui devait, selon un rapport de mission rédigé par Pierre Maes [Maes, 1969], se dérouler en trois phases :

- une phase expérimentale en circuit fermé,

- une phase d'expansion,

- une phase de généralisation progressive permettant d'atteindre en une quinzaine d'années 80 à $85 \%$ de la population d'âge scolaire.

La phase proprement expérimentale, en concomitance avec la préparation de la seconde phase "qui devait porter sur vingt classes dans un rayon d'une vingtaine de kilomètres autour de Niamey » [Maes, 1969, p.1], a concerné deux classes entre janvier et juin 1965. Au cours de la deuxième phase, 730 élèves ont été recrutés pour suivre à la télévision scolaire des programmes de cours d'initiation en première année et de cours préparatoires en deuxième et troisième années. En relation avec l'idée de réduction des coûts et de scolarisation totale rapide, il avait été envisagé de ramener la scolarité primaire à cinq ans au lieu des six années traditionnelles, mais cette idée fut rapidement abandonnée.

Bien évidemment, la conception des programmes, tout autant que celle des matériels pédagogiques et des supports tels que les leçons et documentaires enregistrés, était essentiellement française. Le projet fut en outre financé par la France, que ce soit pour les investissements, la construction des bâtiments, l'achat et la fourniture des appareils de production audiovisuelle, ou les dépenses de personnel et de fonctionnement. Chaque classe était équipée de 
deux téléviseurs qui étaient les véhicules du message pédagogique qui ne passait plus par le maître. L'avantage, tel qu'identifié par les concepteurs et la plupart des observateurs enthousiastes de l'époque, était que le élèves avaient un accès direct aux pédagogues qui avaient conçu les programmes et qui étaient considérés comme évidemment beaucoup plus qualifiés que les maîtres ordinaires. À tort ou à raison, on pensait d'ailleurs que ces derniers, de par la formation qu'ils avaient reçue, étaient nécessairement " aliénés " à la culture française, alors qu'il s'agissait d'assurer aux enfants africains une compréhension du milieu qui était le leur et une intégration harmonieuses dans ce milieu.

\section{De la réussite à l'échec}

La télévision scolaire du Niger a fonctionné de 1964 à 1979, date à laquelle elle fut transformée en une télévision nationale au service de tout le public nigérien. Au moment de sa conception, ses initiateurs en fixaient le seuil de rentabilité à 170000 élèves, avec pour objectif d'obtenir, 15 ans plus tard, un taux de scolarisation de 80 à $85 \%$ [Maes, 1969]. La plupart des rapports et des témoins de l'époque concluent à des résultats éminemment positifs. D'après Pierre Maes [1969, p. 3], pour les autorités nigériennes, " il ne semble pas qu'il $y$ ait de doute sur la supériorité de la télévision par rapport à la pédagogie traditionnelle, pour ce qui est d'apprendre tout simplement à lire, à écrire le français ou à assimiler le programme de calcul et d'arithmétique. L'expérience est donc entièrement positive ». Pour de Saivre [1998, p. 161] : "Ce fut un grand succès, les enfants en deux ans parlaient un excellent français et étaient en avance sur les élèves de l'enseignement traditionnel. [...] Les enfants du CM2 Télévisuel étaient en avance sur ceux de l'enseignement traditionnel ». Pour Egly enfin [1986, p. 339] : "La télévision scolaire du Niger a montré que l'usage intensif de programmes télévisés spécialement conçus permettait, même avec la participation d'enseignants rapidement formés, d'obtenir des résultats très satisfaisants dans toutes les disciplines. »-

II faut ajouter à ces témoignages ceux des principaux concernés : anciens élèves, anciens moniteurs et anciens techniciens. Presque tous nos interviewés admettent que la télévision scolaire nigérienne était une expérience originale dont le succès, selon eux, était indéniable. Par exemple, à la question de savoir s'ils trouvaient l'expérience positive ou négative, sur les trente anciens élèves interrogés, vingt-trois ont répondu positivement ; cinq d'entre eux n'ont donné aucune réponse claire et deux se sont montrés assez nuancés sur les résultats : 
Technologies éducatives et développement:

une brève histoire de la télévision scolaire au Niger

« Ca a été une expérience positive. Par exemple après la fermeture de la télévision scolaire on s'est retrouvés avec deux admis aux examens du CEPE alors qu'avant on avait 42 admis. " (A.D., 44 ans, militaire, promotion 1972-1978).

« Je pense que l'expérience a été positive parce que beaucoup d'anciens élèves de mon village ont eu plus de succès avec la télévision scolaire que ceux qui ne l'ont pas fréquentée. Par exemple, l'élève qui était premier du centre d'examen [où composaient plusieurs centaines d'élèves de différentes écoles] était toujours de la télévision scolaire ». (N.H., 47 ans, outilleur, ancien élève de la télévision scolaire, promotion 1972-1978).

«C'était une expérience positive. Les élèves de la télévision scolaire avaient un niveau supérieur à ceux du système traditionnel ». (G.K., 52 ans, agent d'état civil, ancien élève de la télévision scolaire, promotion 1966-1972).

Ces appréciations sont similaires à celles portées par tous les anciens enseignants eux-mêmes :

«Les élèves réussissaient bien. Sur une classe de 30 élèves présentés au CEPE, au moins 25 réussissaient. » (D.K., 66 ans, enseignant- moniteur de la télévision scolaire à la retraite, 1967-1980).

«Expérience positive. Beaucoup de mes anciens élèves ont réussi dans la vie. » (S. B., 71 ans, ancien directeur de la télévision scolaire, 1965-1978).

Bien entendu, on peut estimer que ces déclarations, parce qu'elles viennent de personnes étroitement liées au système et d'anciens élèves qui sont maintenant cadres de l'administration, sont entachées d'un certain biais lié à leur statut actuel. Ces réactions traduisent néanmoins un sentiment généralement partagé par presque tous ceux qui se sont intéressés à la question, notamment les nombreux évaluateurs et visiteurs de pays étrangers 
désireux de répéter l'expérience chez eux et qui tous ont souligné le taux très élevé de réussite scolaire chez les enfants.

D'ailleurs, même sur le plan socio-pédagogique (c'est-à-dire la prise en compte des facteurs sociologiques, de l'environnement social dans l'approche pédagogique et l'ancrage culturel et social des connaissances transmises en rapport avec le milieu), la télévision scolaire n'a pas été qu'un échec. Contrairement à ce que l'on prétend souvent à propos de la télévision, les élèves n'étaient pas des récepteurs passifs « ingurgitant " des connaissances conçues et délivrées par d'autres. Comme le rapporte Desalmand [1986, p.100], qui a étudié la télévision scolaire en Côte d'Ivoire, ce projet, "contrairement à un discours trop répandu, n'avait pas transformé les enfants en des sujets passifs, obnubilés par l'écran. " II souligne la "spontanéité, la curiosité, l'esprit critique » des élèves passés par la télévision scolaire et estime même que c'est ce qui est à l'origine du divorce entre cette expérience et la société ivoirienne, restée conservatrice et peu encline à accepter ces nouvelles aptitudes développées chez les enfants. La plupart des anciens élèves que nous avons interrogés expriment la même opinion. Pour A. B. S., enseignant de 51 ans, ancien élève de la promotion 1966-1972:

"C'était un enseignement adapté à la réalité
socioculturelle et socio-économique du pays parce
que chaque enseignement est dispensé grâce aux
images de la télé, ensuite exploité par le maître après
l'émission, puis suivi de pratique par les élèves.
Toutes les leçons étaient comme ça ».

Les activités pratiques étaient une composante essentielle de l'enseignement et étaient basées sur l'idée que le savoir-faire était plus important que l'accumulation de connaissances livresques ou la mémorisation de leçons sans rapport avec la réalité. C'est ce que les interviewés traduisent dans leur vocabulaire par la notion "d'enseignement concret » par opposition à ce qui n'est que théorique :

«Les élèves étaient initiés aux travaux manuels: forge, tissage, jardinage, hygiène et assainissement, élevage, reboisement, etc. Des classes-promenades étaient organisées pour découvrir l'environnement. " (H. S., 52 ans, enseignant du secondaire, ancien élève de la télévision scolaire, promotion 1966-1972). 
Technologies éducatives et développement:

une brève histoire de la télévision scolaire au Niger

«La télévision scolaire avait aussi pour ambition de former des agents d'encadrement du monde rural. Le programme prenait en compte les activités pratiques et productives. " (M. A., 52 ans, architecte, ancien élève de la télévision scolaire, promotion 1966-1972).

"La télévision scolaire prenait suffisamment en compte les réalités nationales et culturelles. On nous montrait tout : comment cultiver l'arachide ; comment les gens d'Ingall [ville touarègue du Niger] étaient approvisionnés en céréales. Comment étaient les élèves d'Ingall; on nous apprenait les travaux communautaires, alors qu'on ne connaissait pas tout cela. » (D. T., 59 ans, responsable d'usine, ancien élève de la télévision scolaire, promotion 1972-1978).

"Les chansons et ballets des festivals nigériens nous étaient diffusés. " (Dr. A. B., 46 ans, médecin, ancien élève de la télévision scolaire, promotion 1972-1979).

Les anciens élèves insistent particulièrement sur la connaissance du Niger, c'est-à-dire du milieu, et se montrent plutôt fiers de leur ancrage culturel.

Enfin, une analyse attentive des stratégies mises en œuvre par les promoteurs de l'expérience, des acteurs impliqués et des relations qu'ils ont entretenues les uns avec les autres, des interactions entre acteurs et membres des communautés concernées et de la société globale dans son ensemble révèle qu'il serait trop facile d'attribuer l'échec de ce projet à une approche exclusivement technocentriste ou à la non association des membres de la communauté, à la manière dont procèdent les évaluations fondées sur le critère participatif. Nous verrons plus loin qu'il y avait sans doute une implication insuffisante de la communauté, notamment des parents d'élèves, mais que c'est l'absence de prise en compte effective de leurs contributions qui était en cause et non leur association formelle, car la plupart des anciens élèves et enseignants affirment que les parents étaient associés aux activités pratiques et productives, qu'il y avait des émissions télévisées destinées uniquement aux parents d'élèves, que le chef de village et l'imam (c'est-à-dire l'autorité religieuse) étaient souvent consultés pour toutes les activités programmées, ainsi que les représentants des organisations de jeunesse traditionnelles, appelées à l'époque "Samaria » et implantées dans tous les villages nigériens. Les communautés villageoises ont également contribué, en main-d'œuvre, à la construction des salles de classes et des logements pour les moniteurs. II 
existait aussi des associations de parents d'élèves, tout comme il existait un système de feed-back entre moniteurs, élèves et concepteurs de programmes via un "traveling observer " comme l'appelle Tickton [1971, p. 7] qui visitait régulièrement chaque école et aidait à intégrer les observations ou propositions recueillies. Toutefois, ces points de vue ne sont pas partagés par tous les interviewés et les divergences observées traduisent certainement des orientations personnelles des acteurs à divers moments de la vie de la télévision scolaire qui, il faut le rappeler, a duré quinze ans.

II apparaît, en outre, que le projet n'était pas une expérience uniquement axée sur l'outil technique, mais a concerné aussi les populations. L'époque se prêtait d'ailleurs à cette relative association des communautés villageoises « de base », car l'implantation de la télévision scolaire était elle-même intervenue dans un contexte où le discours développementaliste s'articulait autour de la communication et se traduisait, au niveau des politiques nationales des pays africains, par l'idéologie de la communication pour le développement. Ainsi, par exemple, l'avènement des télévisions scolaires s'est accompagné de la mise en place des radios communautaires qui étaient censées répondre aux besoins d'information et d'éducation des communautés rurales tout en assurant une décentralisation de l'information, la participation des citoyens, l'expression de "la voix des sans voix" (expression qui trouve probablement son origine dans la célèbre citation de l'Abbé Pierre : "Il faut que la voix des hommes sans voix empêche les puissants de dormir »). C'est pourquoi au Niger, parallèlement à la télévision scolaire, les radios communautaires donnaient des cours d'adultes et menaient la "sensibilisation » des populations sur des sujets se rapportant à leur vie quotidienne. La philosophie qui animait les concepteurs du projet était donc aussi participative.

II n'en reste pas moins que l'expérience a été abandonnée et que la télévision scolaire ne forme plus une part intégrante du système éducatif nigérien, ni d'ailleurs des autres pays (Côte d'Ivoire, Sénégal) où des tentatives similaires ont été menées. Selon Duboux [1996, p. 23], " au milieu des années 80, presque toutes ces télévisions scolaires cesseront leurs activités. II aura fallu une dizaine d'années, des milliards de francs et des milliers de pages de rapport pour que les bailleurs de fonds d'abord, les spécialistes enfin, se rendent compte que les télévisions et les écrans n'étaient pas les meilleurs moyens de formation. " Que s'est-il donc passé ? Qu'est-ce qui explique l'écart entre le succès tel que rapporté par les acteurs et l'abandon final du projet? 
Technologies éducatives et développement :

une brève histoire de la télévision scolaire au Niger

\section{Les raisons officielles de l'abandon: contraintes budgétaires et rentabilité financière.}

Pour les décideurs nigériens et les experts français qui les conseillaient, aussi bien que pour les anciens enseignants et élèves (qui pour la plupart, nous l'avons vu, se refusent à parler d'échec), l'abandon du projet fut d'abord motivé par des raisons strictement financières :

« Ne parlons pas d'échec, mais d'abandon à cause du coût extrêmement élevé de la télévision scolaire. » (A. B., enseignant, ancien élève de la télévision scolaire, promotion 1966-1972).

"Il y a eu abandon parce que l'État n'avait pas les moyens de poursuivre l'expérience. » $(\mathrm{H}$. T., 53 ans, enseignant, ancien élève de la télévision scolaire, promotion 1966-1972).

"La télévision scolaire a été abandonnée parce que ça coûtait trop cher. » (S. D., 71 ans, inspecteur d'enseignement à la retraite, ancien directeur d'une école dotée de télévision scolaire).

En effet, la part prise par la télévision scolaire dans les dépenses d'éducation et dans le budget national en général était devenue rapidement insupportable pour le gouvernement nigérien qui devait s'en remettre à la France pour son financement. D'après Carnoy [1975], qui juge les coûts " astronomiques », la mise de fonds de la France pour ce projet a été d'environ 1,5 million de dollars en 1969. Les coûts récurrents ont été de 600000 dollars par an pour la France et d'environ 175000 dollars pour le Niger. Le coût total annuel (y compris les autres sources de financement) était estimé à 925000 dollars ou 1156 dollars par année et par élève; ce qui, bien sûr, était excessif pour l'époque, particulièrement pour un pays sous-développé comme le Niger. D'ailleurs, à mesure que le projet avançait dans le temps, ce caractère insupportable des coûts devint de plus en plus évident, surtout au regard des projections en matière de scolarisation qui visaient à transformer les classes traditionnelles en classes utilisant la télévision scolaire et à atteindre un objectif d'enseignement télévisé de $95 \%$ en 1993 pour un effectif total de 207000 élèves. En outre, le matériel technique exigeait non seulement un entretien mais aussi un 
renouvellement constant, en raison de la recherche d'une plus grande efficacité grâce à des équipements techniques nouveaux et plus performants et souvent aussi plus chers (telles que les batteries solaires en remplacement des batteries alcalines) et cela pour des centaines de classes au Niger. L'objectif de rentabilité n'était donc pas assuré. L'idée que l'enseignement traditionnel était non seulement plus efficient, mais aussi moins coûteux s'imposa peu à peu, contredisant par là même l'objectif premier du projet qui tablait sur une baisse des coûts par élève en raison du fait qu'on pouvait en former un plus grand nombre avec moins d'enseignants et donc moins de moyens financiers.

De plus, le gouvernement se heurta rapidement à un autre problème : dès que la première cohorte des élèves issus de la télévision scolaire entra en sixième, il fallut envisager la poursuite des études en cycle secondaire pour certains d'entre eux qui le réclamaient fortement ; ce qui entraînait des charges croissantes et récurrentes pour des dirigeants politiques confrontés aux coûts élevés de la technologie scolaire. Le rythme d'expansion de l'enseignement primaire et secondaire devint donc rapidement insoutenable. Bref ! La télévision ne paraissait pas rentable en termes économiques. Or, pour faire face à ces problèmes, dont l'origine se trouvait dans le coût de la technologie scolaire, le gouvernement et les experts n'eurent pas d'autre idée que d'envisager leur solution par des moyens... technologiques! On mit au point un projet de faisceau hertzien qui, malheureusement, ne pouvait couvrir qu'une partie limitée du territoire national et laissait même en dehors de la couverture télévisuelle des régions très proches de Niamey, telles que Tillabéry qui n'aurait pu en bénéficier que par la mise en place d'un autre... faisceau hertzien. On envisagea même le lancement d'un satellite de télécommunications qui aurait pu profiter à tous les pays de la sous-région dans un cadre multinational. Bien entendu, tous ces moyens étaient hors de portée du gouvernement nigérien qui devait s'en remettre aux organismes d'aide internationaux et aux organisations financières internationales (telles que la Banque mondiale) pour une réalisation conditionnelle, aléatoire et qui aurait pris du temps pendant que, sous la pression démographique, les besoins en éducation allaient croissant.

Toutefois, comme le projet de télévision scolaire avait été envisagé sous l'angle de la seule rentabilité, le gouvernement aurait pu se satisfaire du fait qu'il pouvait substituer au grand nombre d'instituteurs - qu'il aurait fallu former dans le système traditionnel - un nombre limité de moniteurs dont la formation était beaucoup plus rapide (deux mois), le niveau d'éducation exigé beaucoup plus bas (les moniteurs étaient recrutés parmi ceux qui avaient un certificat d'études) et les coûts évidemment beaucoup moins élevés, du moins au début. Cette approche avait cependant ses limites puisqu'elle supposait d'abandonner à leur sort des diplômés nigériens plus qualifiés pour des raisons uniquement liées à la 
Technologies éducatives et développement :

une brève histoire de la télévision scolaire au Niger

rentabilité. Très vite, la question du niveau de qualification des moniteurs, qui avaient tout juste quelques années d'avance sur leurs élèves, se posa car ils furent l'objet de critiques constantes de la part des parents d'élèves, des instituteurs de l'enseignement classique et des élèves qui sortaient avec un BEPC de l'enseignement secondaire sans trouver de travail là où on employait des gens moins qualifiés qu'eux. Mais recruter des enseignants titulaires du BEPC supposait aussi les payer plus cher, en contradiction flagrante avec l'objectif de rentabilité ; les rémunérer avec un salaire de moniteur n'était pas non plus envisageable. Tous ces facteurs contribuèrent à placer la télévision scolaire sous le feu des critiques, principalement des élèves et enseignants du système traditionnel, mais aussi de certains parents d'élèves :

"Les enseignants du système traditionnel percevaient négativement la télévision scolaire. Pour eux, l'enseignement par la télévision scolaire était de la distraction. » (H. S., 52 ans, enseignant du secondaire, ancien élève de la télévision scolaire, promotion 1966-1972).

« II y avait une sorte de jalousie. Les enseignants du système traditionnel nous considéraient comme des enfants gâtés. » (H. D., 64 ans, enseignant de la télévision scolaire à la retraite, 1966-1980).

«Les enseignants du système classique n'avaient pas de contact avec nous. Ils avaient leur système et nous le nôtre. Ils percevaient négativement l'enseignement télévisuel. Ils considéraient la télévision comme un facteur de perturbation de leur système. " (D. M., moniteur d'enseignement, ancien enseignant à la télévision scolaire, 1965-1974).

La perception courante, au sein des enseignants du système traditionnel, était que le maître était remplacé par l'outil technique, qu'il avait un rôle facile ou qu'il n'avait rien à faire et que donc son salaire ne se justifiait pas. À cela s'ajoutait une dépendance mal perçue par rapport aux techniciens européens (bien que nombre d'entre eux se soient retirés dans les années 1970, remplacés par des technicien nigériens). Le projet avait été conçu par une équipe internationale d'éducateurs, de spécialistes des médias, de psychologues et de sociologues [Tickton, 1971] et employait, comme nous l'avons vu, 55 techniciens français qui étaient payés au taux salarial appliqué en France. En 
outre, il avait fallu faire appel aux services de multiples consultants (toujours européens) chargés de rédiger toutes sortes de rapports et à des visiteurs et experts de toute nature. Toutefois, les raisons de l'abandon sont beaucoup plus complexes qu'un simple coût financier. Elles relèvent de facteurs à la fois politiques et sociaux qui traduisent une confrontation de visions divergentes du monde et de la société par les agents dans leurs rapports à l'objet technique. Au vu de tout ce que nous avons dit plus haut et de ce qui va suivre, on peut en effet estimer que la télévision scolaire était devenue un enjeu qui faisait l'objet d'investissements symboliques par des agents qui occupaient des positions différentes, qui avaient des intérêts et des objectifs différents quant à l'utilisation de ce média.

\section{Visions divergentes du média}

II est clair, par exemple, que ceux qui étaient impliqués dans la conception et la mise en œuvre du projet, tout comme les membres de la société ordinaire nigérienne étaient porteurs de représentations spécifiques de l'objet technique, agissaient en fonction de logiques différentes et tentaient d'imposer leur conception de la télévision scolaire et de ses usages.

Ainsi, on ne peut s'empêcher de remarquer la quasi-unanimité des experts et consultants de tous ordres qui ont visité et étudié l'expérience nigérienne en matière de télévision scolaire et qui, tous, concluaient invariablement à ses aspects positifs là où certains agents de la société nigérienne (parents d'élèves, anciens élèves titulaires du BEPC et enseignants principalement) manifestaient une certaine hostilité au projet. Maes [1969, p. 2] écrit par exemple que "de très nombreux visiteurs venant principalement de France mais aussi de divers pays sont passés au Niger et ont vu fonctionner les télévisions scolaires. De l'avis pratiquement unanime, cette expérience est une révélation extraordinaire de la puissance du petit écran en matière pédagogique [...] Les résultats ont paru tellement positifs [...] que le gouvernement de Côte d'lvoire a décidé de transformer tout son système d'enseignement en vue d'utiliser le plus largement possible la télévision à tous les niveaux. "

Comme nous l'avons vu plus haut, cette unanimité n'était pourtant pas partagée par certains secteurs de la société nigérienne. On peut faire l'hypothèse que cette divergence est le résultat de deux visions opposées de la technologie et de sa finalité, elles-mêmes en relation avec la position respective des agents. À cet égard, le discours unanime des "experts" sur l'impact supposé positif de la télévision scolaire peut même être interprété comme résultant de l'idéologie d'une élite professionnelle désireuse de maintenir les 
Technologies éducatives et développement :

une brève histoire de la télévision scolaire au Niger

avantages associés à l'utilisation de ces objets techniques en Afrique. Cette élite est intéressée à la formulation de politiques nationales de développement et d'éducation qui utilisent ces technologies car elles se traduiraient par plus d'aide étrangère, plus de programmes et de projets avantageux pour eux en termes d'emploi, de consultations et de créations d'ONG. Leur discours serait donc un discours de légitimation de politiques technologiques pour des groupes nationaux et internationaux de professionnels et d'organismes d'aide qui partagent des intérêts communs dans la promotion de ces technologies et dont les visions du monde sont réappropriées par les décideurs et autorités politiques africaines. Ainsi, les représentations de la télévision éducative en Afrique ont été construites à travers un discours qui reflète l'idéologie du développementalisme (fondée sur le déterminisme technologique) et les intérêts de groupes particuliers d'agents.

À l'opposé, les parents d'élèves et les autres membres de la communauté, bien que formellement consultés ou associés, n'avaient pas grand-chose à dire sur l'expérience. Ce qui traduisait surtout l'état d'un certain rapport de forces entre les concepteurs européens du projet, l'État nigérien et les communautés locales. Pour les premiers, la télévision était un outil qui devait changer non seulement la façon d'apprendre mais surtout rendre efficace l'enseignement sous son angle quantitatif essentiellement (combien d'élèves formés en combien de temps et à quels coûts). C'est sous le prisme de l'objet technique qu'étaient appréhendés le système éducatif et ses effets sur la société d'ensemble. Cette approche technocratique se traduisait par l'obsession de la rentabilité au sens quantitatif du terme et l'impact que l'introduction de cette technologie devait avoir sur le reste de la société, sa capacité à la transformer, pour ainsi dire, de l'extérieur comme dans un espèce de causalité linéaire qui devait conduire de l'objet technique aux élèves puis à la société.

Pour les seconds, l'acceptation de l'école moderne elle-même, en tant que système d'éducation des enfants, posait problème. II faut encore une fois rappeler que le pays sortait tout juste de la colonisation, qui était à la fois un système de domination économique, politique et culturel. L'école était encore perçue comme une menace sur les valeurs culturelles traditionnelles :

«À cette époque, nos parents en général percevaient négativement la télévision scolaire et l'école tout court. Pour eux, c'était l'école des Blancs, l'école amenée par les colons, donc imposée. " (M. S., 52 ans, agent d'hygiène, ancien élève de la télévision scolaire, promotion 1966-1972). 
« Nos parents percevaient négativement la télévision scolaire. Ils y étaient hostiles. " (A. B. S., 51 ans, enseignant, ancien élève de la télévision scolaire, promotion 1966-1972).

« La télévision scolaire était perçue négativement car nos parents ne voulaient même pas de l'école surtout que les premières classes étaient implantées en plein milieu rural, au sein de nos communautés et qu'elles apparaissaient comme une menace directe sur les valeurs sociales et culturelles. " $(H$. S., 52 ans, enseignant du secondaire, ancien élève de la télévision scolaire, promotion 1966-1972).

" À cette époque-là, il y avait un conservatisme traditionnel et islamique [rappelons que le Niger est musulman à plus de $98 \%$ ] très prononcé. L'école en général était considérée comme un reniement des valeurs de la société. » (O. I., 50 ans, technicientranscripteur, ancien élève de la télévision scolaire, promotion 1966-1972).

On voit donc que ce qui était perçu par les promoteurs européens comme une démarche progressiste et un souci de prise en compte du milieu de l'élève apparaissait au contraire pour beaucoup de parents comme une menace pour leurs valeurs socioculturelles. Toutefois, ces derniers n'échappaient pas à la fascination exercée par le petit écran dans ces zones où, à l'époque, la télévision était encore à peine connue. Comme le souligne l'un de nos interlocuteurs, "ils voyaient comme un spectacle fantastique les émissions montrées aux élèves » et ressentaient donc une certaine fierté à l'idée que leurs enfants faisaient partie de ce monde nouveau. II n'en demeurait pas moins que l'école, présentée comme obligatoire par les autorités politiques, apparaissait comme imposée à des populations impuissantes qui ne faisaient ce qu'on leur demandait (comme de suivre par exemple les émissions de "sensibilisation " diffusées chaque vendredi) que par obéissance à l'autorité. Le contexte de l'époque, qui était encore celui des régimes politiques dictatoriaux d'avant la vague des démocratisations des années 1990, favorisait largement la perception de la télévision comme un instrument au service des dominants - et ceux-ci allaient d'ailleurs rapidement pressentir le profit qu'ils pouvaient en tirer comme instrument de contrôle des populations. Nous avons insisté sur le fait que la télévision scolaire n'était pas un échec et précisé que les raisons 
Technologies éducatives et développement :

une brève histoire de la télévision scolaire au Niger

officielles de son abandon étaient financières, mais on peut aussi constater que la décision des autorités a été motivée par des raisons politiques.

Pour s'en convaincre, il suffit de rappeler l'attitude du régime de Seyni Kountché à l'égard du projet. En effet, en 1974 (soit dix ans après le début de l'expérience), le gouvernement du Président Diori Hamani sera renversé par le colonel Seyni Kountché qui instaurera un régime dictatorial jusqu'à sa mort en 1987. L'une des caractéristiques principales de son régime était l'embrigadement des populations urbaines et rurales pour des objectifs de "construction nationale » et le contrôle strict des médias. Les seuls tolérés étaient les médias officiels, propriété de l'État: l'organe de radiodiffusion nationale ORTN (Office de Radio-Diffusion Télévision du Niger), le journal officiel gouvernemental Le Sahel et, plus tard, Télé Sahel, télévision nationale sous contrôle gouvernemental à l'origine de laquelle on trouve Télé-Niger, c'està-dire la télévision scolaire. Si donc quatre ans après son arrivée au pouvoir, Kountché a décidé d'abandonner la télévision scolaire, c'était aussi parce qu'il voulait s'approprier des infrastructures, des équipements et des techniciens formés en grand nombre pour les mettre au service de son régime ; en effet, la télévision scolaire qui fut directement transformée en télévision nationale à partir de 1978. Et, de fait, cette nouvelle télévision va devenir l'un des principaux instruments de propagande et de contrôle des populations sous le régime de Seyni Kountché qui avait donc des objectifs différents de ceux de Diori Hamani. II sut ainsi tirer profit du coût financier exorbitant du projet, de l'hostilité développée par une frange des enseignants exerçant dans le système traditionnel et par de nouveaux diplômés du système d'enseignement traditionnel, ainsi que de l'hostilité latente des populations nigériennes en général. Kountché va alors transformer la télévision scolaire, non seulement en une télévision nationale, mais en "télévision pour le développement", réorientant le rôle assigné à ce média en conformité avec l'idéologie et les attentes du régime. Cela vient conforter l'idée d'un usage des technologies commandé par les rapports sociaux et politiques, à l'inverse des présupposés qui font dépendre l'évolution sociale de l'introduction de nouvelles technologies.

\section{Conclusion}

Au cours des récentes années, de multiples expériences éducatives, toutes centrées autour des TIC, ont vu le jour en Afrique. Ce sont, par exemple, les écoles numériques de brousse, les expériences d'alphabétisation par téléphonie cellulaire, les universités virtuelles africaine ou francophone et les campus 
numériques francophones précédemment évoqués. Dans beaucoup de cas, l'idée qui sous-tend ces expériences est que les TIC ont la capacité de pallier l'absence d'infrastructures éducatives, le manque de personnel et de compétences, le non accès à la documentation scientifique et technique spécialisée et de réaliser un bond de géant vers le développement. Néanmoins, ce n'est pas la seule fois que des projets grandioses et des espoirs de développement accéléré ont été fondés sur l'usage et l'adoption de technologies nouvelles auxquelles on accordait la capacité de faire des miracles. La télévision scolaire du Niger a été une de ces expériences. Elle trouve son origine dans des projets similaires implantés en France, puis transposés en Afrique. Si elle a été abandonnée cependant, ce n'est pas nécessairement, ainsi que nous nous sommes efforcé de le montrer, parce qu'elle était utopique ou a échoué. Le contexte sociopolitique de sa mise en œuvre permet d'expliquer les problèmes qui allaient mener à son abandon. La télévision a en effet été un enjeu de lutte entre divers agents sociaux, tous dotés de représentations divergentes de l'outil technique et tous mobilisés pour imposer leur vision de la technologie et de son usage. La transformation finale de la télévision scolaire en télévision nationale tournée vers des objectifs de "construction nationale ", de "développement" et de "sensibilisation des populations" traduit l'issue de cette lutte.

\section{Références bibliographiques}

CARNOY M. 1975, "The Economic Costs and Returns to Educational Television”, Economic Development and Cultural Change, vol. 23, n ${ }^{\circ} 2$, pp. 207-248.

DESALMAND P. 1986, « Une aventure ambiguë : le programme d'éducation télévisuelle (1971-1982) », Politique africaine, $n{ }^{\circ} 24$, pp. 91-103.

DUBOUX R. 1996, « De la télévision scolaire à la culture multimédia », Communication et langages, $n^{\circ} 110$, pp. 20-34.

EGLY M. 1973, « L'image pédagogique à Télé-Niger », Communication et langages, $n{ }^{\circ} 18$, pp. 31-45.

EGLY M. 1979, «Les utopies éducatives audio-visuelles », Communication et langages, vol. $41, n{ }^{\circ} 41-42$, pp. 53-69.

EGLY M. 1984. Télévision didactique : entre le kitsch et les systèmes du troisième type ? Paris : Edilig. 
Technologies éducatives et développement:

une brève histoire de la télévision scolaire au Niger

EGLY M. 1986, « L'utilisation de la télévision scolaire au Niger, en Côte d'Ivoire et au Sénégal », Revue Internationale de l'éducation, vol. 32, ${ }^{\circ}{ }^{\circ} 3$, pp. 338346.

GLIKMAN, V. 1995. "Les avatars de la télévision éducative pour adultes en France : histoire d'une "non-politique" (1964-1985) », Revue française de pédagogie, vol. 110, pp. 63-74.

LYBERT, T.J., ACKER, J. et KSOLL, C., 2010, « ABC, 123. The Impact of a Mobile Phone literacy Program on Educational Outcomes », working paper 223, Center for Global Development.

MAES P. 1969, Les perspectives d'extension de la télévision scolaire au Niger, rapport de mission (5-25 juin 1969), Niamey : ministère de l'Éducation.

MARIE A.L. 2009, "Un ordinateur dans la classe, pourquoi faire? »

<http://www.rfi.fr/actufr/articles/110/article_78748.asp>, dernière consultation le 5 mars 2011.

MAYO J. K., 1976, Educational reform with television: the El Salvador experience, Stanford (California) : Stanford University Press

SAIVRE de, D. 1998, « 20, Rue Monsieur. Pédagogie et coopération », Politique Africaine $n^{\circ} 72$, pp.159-185.

TICKTON, S. G. 1971, « Recent Developments in Instructional Technology in the Developing World », Paper presented at the Educational Technology Workshop of the Council for Higher Education in the American republics, Salvador, Brazil, May 26-29.

VINCENT G. 1981, "Pour une méthode d'analyse des phénomènes scolaires ", in : P. CASPARD, S. CHASSAGNE, Y. LEQUIN, J. OZOUF et A. PROST

(dir.), Cent ans d'école, Seyssel : Éditions Champ Vallon 